\title{
Inhalt (Contents), Vol. 1, No. 8, 1973-74
}

\section{Impressum (Imprint) 583}

Sonderhefte zu »Die Infusionstherapie« (Special Issues of “Die Infusionstherapie”)

Ankündigung des redaktionellen Programms (Editorial Program) 587

Symposion »Parenterale Ernährung« (Symposium "Parenteral Nutrition”) 589-613

Quirin, H., Schaeffer, G. und Kluthe, R., Freiburg

Stickstoffbilanz bei parenteraler Zufuhr von Aminosäurenlösungen (Nitrogen Balance during

Parenteral Administration of

Amino Acid Solutions) 589

Jekat, F., Bochum

Zur Stöchiometrie und ernährungsphysiologischen Bedeutung von

Aminosäureninfusionslösungen (Stoichiometry and

Nutritional-Physiological Significance of Amino Acid Infusion Solutions) 594

JÜRGENS, P. und DOLIF, D., Hamburg

Über den Aminosäurenbedarf Erwachsener unter den Bedingungen der parenteralen Ernährung (Amino Acid Require

ments of Adults under the Condition of Parenteral Nutrition)

603

Diskussion (Discussion) 611

Dölp, R., Bauer, H., Ahnefeld, F. W. und Seeling, W., Ulm

Klinische Untersuchungen über die routinemäßige Infusionstherapie mit 1,5\%igen

Aminosäurenlösungen in der operativen

Medizin (Clinical Studies on the Routine Infusion Therapy with 1.5\% Amino Acid Solutions in Surgery) $\quad 615$

Lundsgaard-Hansen, P. und Pappova, E., Bern

Respiratorische Insuffizienz, kolloidosmotischer Druck und Albumintherapie (Respiratory

Failure, Colloid Osmotic Pres

sure, and Albumin Treatment)

624

Busch, H., von Eisenhart-Rothe, B. und Sasse, U. K., Hamburg

Hepatitis B und Blutübertragung - »Transfusionshepatitis« oder »Hospitalhepatitis«? (Type-B

Hepatitis and Blood

Transfusion - “Transfusion-Associated Hepatitis” or "Hospital-Acquired Hepatitis”?)

Goldfield, M, Trenton

Some Epidemiologic Studies of Transfusion-Associated Hepatitis (Einige epidemiologische Untersuchungen über Hepatitis

in Zusammenhang mit Transfusionen) 645

Tschirren, B., Affolter, U, Elsässer, R., Freihofer, U. A., Grawehr, R., Müller, P. H. und Lundsgaard-Hansen, P., Bern

Der klinische Plasmaersatz mit Gelatine: Zwölf Jahre Erfahrungen mit 39320 Einheiten Physiogel ${ }^{\circledR}$ (The Clinical Plasma

Substitution by Gelatin: Twelve Years of Experience with 39,320 Units of Physiogel ${ }^{\circledR}$ ) 
Buchbesprechungen (Book Reviews)

Falser, N., Innsbruck

Serologische Untersuchungen an Blutspendern: III. Erfassung einer »Leberschädigung« im Blut des Spenders (Serological

Examinations in Blood Donors: III. Detection of a "Liver Damage" in the Donor Blood) 666 Autorenverzeichnis (Authors'Index) 671

Hinweise für Autoren (Instructions to Authors)

672

Gesamtinhaltsverzeichnis (Contents Volume 1)

Zum Titel

Die acht auf der Titelseite aufgeführten Themengruppen stellen das redaktionelle Programm des ersten Bandes dieser Zeit-schrift dar. Bei jenen Themengruppen, zu denen im Heft Arbeiten erscheinen, ist der vorangesetzte Kreis ausgefüllt.

(C) Copyright 1974 by S. Karger Verlag GmbH, Verlag für Medizin und Naturwissenschaften, D8034 Germering, Postfach 2 